\title{
Elevated CD133, but not VEGF or EGFR, as a predictive marker of distant recurrence after preoperative chemoradiotherapy in rectal cancer
}

\author{
HIROMI YASUDA $^{1}$, KOJI TANAKA ${ }^{1}$, SUSUMU SAIGUSA $^{1}$, YUJI TOIYAMA ${ }^{1}$, \\ YUHKI KOIKE $^{1}$, YOSHINAGA OKUGAWA ${ }^{1}$, TAKESHI YOKOE ${ }^{1}$, AYA KAWAMOTO ${ }^{1}$, \\ YASUHIRO INOUE $^{1}$, CHIKAO MIKI ${ }^{1}$ and MASATO KUSUNOKI ${ }^{1,2}$
}

\begin{abstract}
Departments of ${ }^{1}$ Gastrointestinal and Pediatric Surgery and ${ }^{2}$ Innovative Surgery, Division of Reparative Medicine, Institute of Life Sciences, Mie University Graduate School of Medicine, Edobashi 2-174, Tsu, Mie 514-8507, Japan
\end{abstract}

Received March 16, 2009; Accepted May 4, 2009

DOI: 10.3892/or_00000491

\begin{abstract}
CD133 has been postulated to be a colon cancer stem cell (CSCs) marker. Recent investigations suggest that CSCs might contribute to cancer recurrence and resistance to conventional therapies. This study aimed to evaluate the role of CD133 in residual cancer cells after chemoradiotherapy (CRT) for rectal cancer. Forty patients with rectal cancer underwent CRT followed by surgery. Total RNAs of rectal cancer cells before $(n=30)$ and after $(n=40)$ CRT were isolated. Intratumoral CD133, vascular endothelial growth factor (VEGF), and epidermal growth factor receptor (EGFR) levels were measured using real-time reverse transcription polymerase chain reaction. Immunohistochemical staining of CD133 after CRT was also investigated. CD133 in residual cancer cells was higher than in stromal cells in post-CRT specimens $(\mathrm{p}<0.0001)$. The levels of CD133 were found to have increased in post-CRT specimens $(\mathrm{p}=0.0184)$, while VEGF and EGFR levels decreased during CRT $(\mathrm{p}<0.0001$ and $\mathrm{p}=0.0002$, respectively). Patients who developed distant recurrence had a higher post-CRT CD133 compared with those patients without recurrence $(\mathrm{p}=0.0136)$. Elevated postCRT CD133 was associated with poor disease-free survival
\end{abstract}

Correspondence to: Dr Koji Tanaka, Department of Gastrointestinal and Pediatric Surgery, and Innovative Surgery, Division of Reparative Medicine, Institute of Life Sciences, Mie University Graduate School of Medicine, 2-174 Edobashi, Tsu, Mie 514-8507, Japan

E-mail: qouji@clin.medic.mie-u.ac.jp

Abbreviations: VEGF, vascular endothelial growth factor; EGFR, epidermal growth factor receptor; RT-PCR, reverse transcriptionpolymerase chain reaction

Key words: CD133, vascular endothelial growth factor, epidermal growth factor receptor, chemoradiotherapy, rectal cancer $(\mathrm{p}=0.0168)$. Immunohistochemical staining of the cytoplasmic and apical/endoluminal membranous CD133 was observed in residual cancer cells after CRT. CD133 expression in residual cancer cells after CRT may indicate a treatment resistant phenotype in putative CSCs. Elevated CD133, but not VEGF or EGFR, on FFPE specimens may be a predictive marker of distant recurrence and poor survival after preoperative CRT in rectal cancer.

\section{Introduction}

Rectal cancer is one of the most common gastrointestinal cancers in Japan as well as in the Western world. Introduction of chemoradiotherapy (CRT) and total mesorectal excision (TME) has improved sphincter preservation rate, local pelvic control, and survival (1). Despite these significant improvements, distant recurrence remains the major cause of mortality in rectal cancer patients treated with preoperative CRT followed by TME (2).

A rare tumor subpopulation called cancer stem cells (CSCs) or tumor initiating cells (TICs) have been identified and proven in functional assays of stem/progenitor cell properties including the ability to self-renew, differentiate, and proliferate $(3,4)$. CSCs appear to not only be responsible for tumorigenic capability but also confer resistance to chemotherapy or radiotherapy $(5,6)$. Conventional cytotoxic therapies including CRT initially shrink the bulk of a tumor, but fail to eradicate it, resulting inevitably in cancer recurrence. Accordingly, we hypothesized that CRT decreased nonCSCs which are sensitive to CRT, while it may increase the percentage of putative CSCs characteristic of CRT resistance in the population of residual cancer cells.

CD133 (known as prominim-1) is regarded as one of the most important markers of colon CSCs by two studies reporting that CD133 expressing cells sorted by fluorescenceactivated cell sorting system have shown tumor-forming abilities in xenografts $(7,8)$. Pancreatic CSCs with CD133 expression demonstrated a higher metastatic potential compared with those without it (9). Stem cell-like glioma cells with CD133 expression promoted tumor angiogenesis 
through the vascular endothelial growth factor (10). Accumulating evidence indicates that CSCs are associated with tumor metastasis and angiogenesis. Furthermore, CD133 has been reported to be associated with metastatic potential and poor survival in certain human malignancies $(11,12)$.

Vascular endothelial growth factor (VEGF) is intimately involved in the regulation of tumor-associated angiogenesis and has been reported to be associated with tumor aggressiveness, metastasis and poor prognosis (13). Epidermal growth factor receptor (EGFR) is a cell membrane tyrosine kinase receptor which initiates a complex intracellular signal transduction cascade that promotes cancer cell division, migration, angiogenesis, and apoptosis inhibition. EGFR has been also reported to be associated with increased risk of metastasis and poor survival (14).

The VEGF and EGFR pathways have shown to be closely related to each other and share common downstream signaling. Recent preclinical and clinical studies demonstrate that combining VEGF and EGFR inhibition is a feasible therapeutic approach and provides promising efficacy in a range of solid tumors including colorectal cancer $(15,16)$.

First, to clarify the potential association between CRT resistance and the existence of putative CSCs, CD133 expression was investigated in rectal cancer by using preCRT tumor biopsies and post-CRT micro-dissected residual cancer cells. Second, to examine the association between CD133 expression and tumor angiogenesis, correlation between CD133 and tumor angiogenesis-related VEGF and EGFR were also investigated during CRT. We also evaluated the association between post-CRT CD133 expression, clinicopathological variables, and survival.

\section{Materials and methods}

Patients. A total of 40 patients with locally advanced rectal cancer were included in the current analyses. All patients were treated with preoperative chemoradiotherapy (CRT) followed by surgery at the Department of Gastrointestinal and Pediatric Surgery in the Mie University Graduate School of Medicine. Selection criteria were the availability of the quality of isolated RNA for real-time PCR with complete clinical data.

Treatment. The treatment included pelvic radiotherapy using a four-field box technique and concomitant chemotherapy consisting of 5-FU given as $600 \mathrm{mg} / \mathrm{m}^{2}$ administered intravenously for $24 \mathrm{~h}$ and UFT (Tegafur and Uracil) given as $400 \mathrm{mg} /$ body weight administered orally for 5 days a week.

Preoperative radiotherapy was delivered to the whole pelvis at a dose of $20 \mathrm{~Gy}$ in 4 fractions ( 5 Gy per fraction) a week. All patients underwent computed tomography (CT) simulation for three-dimensional radiotherapy planning and were treated with a $10 \mathrm{MV}$ photon using a linear accelerator. The radiation field encompassed a volume that included the primary tumor, mesorectum, presacral space, whole of the sacral hollow, and regional lymph nodes. The superior border was placed at L5/S1 and the inferior border at $3 \mathrm{~cm}$ or more caudal to the primary tumor. Rectal resection with TME was performed within 2 weeks of the end of CRT.
Pathological evaluation and treatment response. Pathological evaluation in the resection specimens was performed according to TNM classification (17). Tumor regression of the primary tumor was semi-quantitatively determined by the amount of viable tumor vs. the amount of fibrosis. This ranged from no evidence of any treatment effect to a complete response with no viable tumor identified, as described by Dworak et al (18). Tumor regression grade (TRG) 0 was defined as no regression; TRG 1 , minor regression (dominant tumor with fibrosis in $\leq 25 \%$ of the tumor mass); TRG 2, moderate regression (dominant tumor with fibrosis in $26-50 \%$ of the tumor mass); TRG 3, good regression ( $>50 \%$ tumor regression); and TRG 4 , total regression (no viable tumor cells, only fibrotic mass).

Microdissection in formalin-fixed, paraffin-embedded (FFPE) specimens. Tumor specimens were fixed in $10 \%$ formaldehyde solution and embedded in paraffin. Sections (10- $\mu \mathrm{m}$ thick) of FFPE specimens were stained with nuclear fast red and subsequently manually microdissected to collect residual cancer and stromal cells, with reference to hematoxylin and eosin sections.

RNA extraction from FFPE specimens. Microdissected samples were digested with proteinase $\mathrm{K}$ in lysis buffer containing Tris-HCl, EDTA, and sodium dodecyl sulfate as previously reported, with minor modifications (19). RNA was purified by phenol and chloroform extraction.

cDNA synthesis. cDNA was synthesized with random hexamer primers and Superscript III reverse transcriptase (Invitrogen, Carlsbad, CA), according to the manufacturer's instructions.

Real-time quantitative RT-PCR. Real-time quantitative RT-PCR analysis was done with the SYBR-Green PCR Master Mix using an Applied Biosystems 7500 Real-Time PCR System according to the manufacturer's instructions (Applied Biosystems, Inc., Foster City, CA). Primers and probes for CD133, VEGF, EGFR and $\beta$-actin were designed with Primer3 software (Biology Workbench Version 3.2, San Diego Supercomputer Center, at the University of California, San Diego, CA). Sequences are shown in Table I. PCR was performed in a final volume of $25 \mu \mathrm{l}$ with a SYBR-Green PCR Master Mix using $1 \mu \mathrm{l} \mathrm{cDNA}, 400 \mathrm{nM}$ of each primer for the respective genes. Cycling conditions were $50^{\circ} \mathrm{C}$ for $2 \mathrm{~min}$ and $95^{\circ} \mathrm{C}$ for $10 \mathrm{~min}$ followed by 40 cycles at $95^{\circ} \mathrm{C}$ for $15 \mathrm{sec}$ and $60^{\circ} \mathrm{C}$ for $1 \mathrm{~min}$.

Relative mRNA levels of target genes. Relative mRNA levels were determined by the standard curve method. The standard curves and line equations were generated using 5-fold serially diluted solutions of cDNA from the colon cancer cell line LoVo. All standard curves were linear in the analyzed range with an acceptable correlation coefficient (R2). The level of target gene expression was calculated from the standard curve. Quantitative normalization of cDNA in each sample was performed using the expression of the B-actin gene as an internal control. Finally, mRNA levels of the target gene were given as ratios to $\beta$-actin mRNA levels. Real- 
Table I. Primer and probe sequences of target genes.

\begin{tabular}{lll}
\hline Gene & Primer and probe & Sequence \\
\hline CD133 & Forward primer & 5'-GCTTTGCAATCTCCCTGTTG-3' \\
& Reverse primer & 5'-TTGATCCGGGTTCTTACCTG-3' \\
VEGF & Forward primer & 5'-CAGAAGGAGGAGGGCAGAA-3' \\
& Reverse primer & 5'-CTCGATTGGATGGCAGTAGC-3' \\
EGFR & Forward primer & 5'-CCTATGTGCAGAGGAATTATGATCTTT-3' \\
& Reverse primer & 5'-CCACTGTGTTGAGGGCAATG-3' \\
ß-actin & Forward primer & 5'-ACAGAGCCTCGCCTTTGC-3' \\
& Reverse primer & 5'-GCGGCGATATCATCATCC-3' \\
\hline
\end{tabular}

time PCR assays were done in duplicate for each sample and mean values were used for calculations of the mRNA levels.

Immunohistochemistry for CD133 in rectal cancer after CRT. Sections $(2-\mu \mathrm{m}$ thick) of FFPE specimens were made. After deparaffinization and dehydration for antigen unmasking, specimens were brought to a boil in $10 \mathrm{mM}$ sodium citrate buffer. Specimens were then blocked and incubated with primary antibody overnight at $4^{\circ} \mathrm{C}$. The antibody was detected by Envision reagents (Envision kit/HRP, Dako Cytomation, Denmark). All sections were counterstained with hematoxylin. Primary CD133 rabbit monoclonal antibody (Cell Signaling Technology) was used in a dilution of 1:100 with labeled streptavidin-biotin method (LASB2 kit/HRP, Dako) added. Negative controls were run simultaneously with pre-immune immunoglobulin.

Statistical analysis. All statistical analyses were performed using JMP version 5 (SAS Institute Inc., Cary, NC, USA). The value of each target gene is expressed as median value (inter-quartile range).

Associations between gene expression levels (continuous variables) and clinicopathological variables (categorical variables) were evaluated using Mann-Whitney U test for two groups or Kruskal-Wallis test for multiple groups.

Disease-free survival was calculated from the date of surgery to the date of disease recurrence. Overall survival was calculated from the date of surgery to the date of death due to rectal cancer or last follow-up. Survival was evaluated using the Kaplan-Meier method. The log-rank test was used to compare the cumulative survival durations in the patient groups.

A non-parametric receiver operating characteristic (ROC) analysis was performed to calculate the best cut-off values predictive of distant recurrence using Medcalc 7.2 for Windows (Mariakerke, Belgium). P-values of $<0.05$ were considered statistically significant.

\section{Results}

Patient characteristics and survival. Forty patients were included in this study. The median age was 65 years (range
37-78 years); the male to female ratio was $3.4: 1$. The postCRT pathological T stages were pT1 (10\%), pT2 (25\%), pT3 $(62.5 \%)$, and pT4 (2.5\%), respectively. Thirteen patients $(32.5 \%)$ had pathological lymph node metastases. Lymphatic invasion was present in 31 of 40 patients $(77.5 \%)$, and vascular invasion was present in 26 patients $(65 \%)$, respectively. Thirty-four tumors $(85 \%)$ showed well- or moderately-differentiated adenocarcinoma histology. None of the patients had local recurrence. Patterns of distant recurrence include liver and lung metastases in 2 patients, lung metastases alone in 3 patients, and peritoneal metastasis in one patient.

Medians of overall survival (OS) and disease-free survival (DFS) were 38.1 months (range; 6.8-86.3) and 41.3 months (range; 2.5-86.3), respectively. The presence of distant recurrence and pathological stage were significantly associated with OS ( $\mathrm{p}=0.0018$ and $\mathrm{p}=0.0174$, respectively). Age over 65 years and pathological positive lymph nodes were significantly associated with DFS ( $\mathrm{p}=0.0486$ and $\mathrm{p}=0.0469$, respectively). The other clinical parameters were not associated with OS or DFS.

Pathological response to chemoradiotherapy. The results of TRG are summarized in Table II. TRG was as follows: TRG 0, 0 patient (0\%); TRG 1, 7 patients (17.5\%); TRG 2, 17 patients (42.5\%); TRG 3, 16 patients (40\%) and TRG 4, 0 patient $(0 \%)$. No TRG 4 (complete regression of the primary tumor) was seen in this study because of unavailability of residual cancer cells. Tumor or node downstaging was demonstrated in 23 patients $(57.5 \%)$.

Correlations of CD133, VEGF and EGFR in pre- and post$C R T$ specimens. To clarify the role of CD133 expression in rectal cancer, correlations of CD133, VEGF and EGFR were examined in pre-CRT tumor biopsy specimens $(n=30)$ and post-CRT FFPE specimens $(n=40)$. There were significant positive correlations between CD133 and VEGF $(\mathrm{r}=0.378, \mathrm{p}=0.0392)$, between CD133 and EGFR ( $\mathrm{r}=0.774$, $\mathrm{p}<0.0001)$ or between VEGF and EGFR $(r=0.390 \mathrm{p}<0.0332)$ in pre-CRT tumor biopsy specimens (Fig. 1). However, these correlations were not observed in post-CRT FFPE specimens (Fig. 2). 
Table II. Association of post-CRT CD133, VEGF, and EGFR with clinicopathological variables.

\begin{tabular}{|c|c|c|c|c|c|c|c|}
\hline Variable & No. & CD133 & p-value & VEGF & p-value & EGFR & $\mathrm{p}$-value \\
\hline \multicolumn{8}{|l|}{ Gender } \\
\hline Male & 31 & 0.183 & & 0.029 & & 0.014 & \\
\hline Female & 9 & 0.115 & 0.55 & 0.038 & 0.07 & 0.010 & 0.94 \\
\hline \multicolumn{8}{|c|}{ Age (median, 65 years) } \\
\hline$\leq 65$ & 21 & 0.174 & & 0.033 & & 0.014 & \\
\hline$>65$ & 19 & 0.183 & 0.51 & 0.031 & 0.75 & 0.013 & 0.36 \\
\hline \multicolumn{8}{|c|}{ Pathological T category } \\
\hline $\mathrm{T} 1$ & 4 & 0.123 & & 0.039 & & 0.027 & \\
\hline $\mathrm{T} 2$ & 10 & 0.119 & & 0.027 & & 0.012 & \\
\hline $\mathrm{T} 3$ & 25 & 0.219 & & 0.031 & & 0.012 & \\
\hline $\mathrm{T} 4$ & 1 & 0.278 & 0.22 & 0.092 & 0.78 & 0.021 & 0.52 \\
\hline \multicolumn{8}{|c|}{ Pathological N category } \\
\hline Present & 13 & 0.295 & & 0.03 & & 0.434 & \\
\hline Absent & 27 & 0.139 & 0.32 & 0.032 & 0.59 & 0.274 & 0.90 \\
\hline \multicolumn{8}{|l|}{ Histology } \\
\hline Well-moderate & 34 & 0.179 & & 0.032 & & 0.015 & \\
\hline Poor/muc. & 6 & 0.196 & 0.94 & 0.022 & 0.12 & 0.010 & 0.17 \\
\hline \multicolumn{8}{|c|}{ Lymphatic invasion } \\
\hline Present & 31 & 0.21 & & 0.032 & & 0.013 & \\
\hline Absent & 9 & 0.107 & 0.32 & 0.029 & 1.00 & 0.012 & 0.53 \\
\hline \multicolumn{8}{|l|}{ Vascular invasion } \\
\hline Present & 26 & 0.286 & & 0.032 & & 0.013 & \\
\hline Absent & 14 & 0.097 & 0.07 & 0.031 & 0.49 & 0.015 & 0.41 \\
\hline \multicolumn{8}{|c|}{ Tumor regression grading } \\
\hline TRG 1 & 7 & 0.139 & & 0.025 & & 0.015 & \\
\hline TRG 2 & 17 & 0.183 & & 0.03 & & 0.014 & \\
\hline TRG 3 & 16 & 0.197 & 0.99 & 0.032 & 0.96 & 0.012 & 0.64 \\
\hline
\end{tabular}

Expression of CD133, VEGF, and EGFR in matched preand post-CRT specimens. To confirm whether expression of CD133, VEGF, and EGFR were altered during CRT, the expression of each gene was compared between matched pre-CRT and post-CRT specimens (Table IV). CD133 was significantly increased in post-CRT specimens ( $\mathrm{p}=0.0184)$, suggesting the selection of putative CSCs during CRT. In contrast, VEGF and EGFR were significantly decreased during CRT ( $p<0.0001$ and $\mathrm{p}=0.0002$, respectively).

Post-CRT CD133, VEGF, and EGFR levels in residual rectal cancer and stromal cells. Median value of post-CRT CD133 level was 0.179 (inter-quartile range, 0.051-0.563) in residual cancer cells, whereas it was only 0.003 (inter-quartile range, 0.001-0.009) in surrounding stromal cells. Post-CRT CD133 and VEGF were significantly higher in residual cancer cells compared with those in stromal cells $(\mathrm{p}<0.0001$ and $\mathrm{p}=0.0002$, respectively, Fig. 3). No significant difference in post-CRT EGFR levels were found between residual cancer and stromal cells.
Associations of post-CRT CD133, VEGF, and EGFR with clinicopathological variables. Median value of post-CRT CD133, VEGF and EGFR mRNA levels were 0.179 (interquartile range; 0.051-0.563), 0.032 (0.018-0.040), and 0.013 (0.009-0.020), respectively.

Associations between post-CRT CD133, VEGF and EGFR and clinicopathological variables are summarized in Table II. No significant associations of post-CRT CD133, VEGF and EGFR with clinicopathological variables were observed.

As shown in Table III, patients who developed distant recurrence $(n=6)$ had significantly higher postCRT CD133 levels compared with those patients without recurrence $(n=34)(p=0.0136)$. No significant association between distant recurrence and VEGF or EGFR was observed.

Predictive value of post-CRT CD133 for distant recurrence and disease-free survival. On the basis of these results, receiver operating characteristic curve (ROC) analyses were 
Correlations of CD133, VEGF and EGFR in pre·CRT tumor biopsy specimens
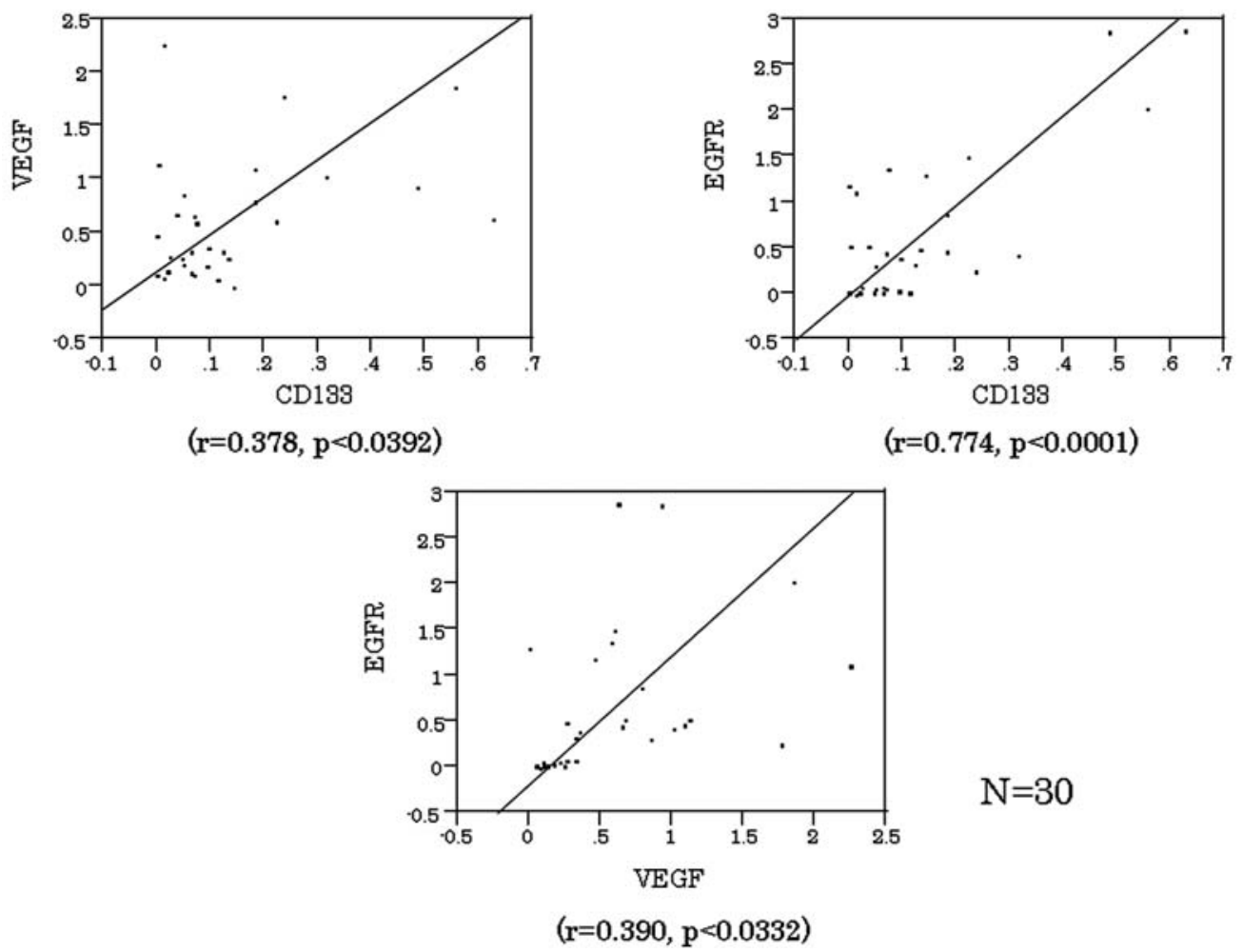

Figure 1. Correlations of CD133, VEGF and EGFR in pre-CRT tumor biopsy specimens. There were significant positive correlations between CD133 and VEGF ( $r=0.378, p=0.0392$ ), between CD133 and EGFR ( $r=0.774, p<0.0001)$ or between VEGF and EGFR ( $r=0.390$ p $<0.0332$ ) in pre-CRT tumor biopsy specimens.

Correlations of CD133, VEGF and EGFR in post·CRT residual cancer cells
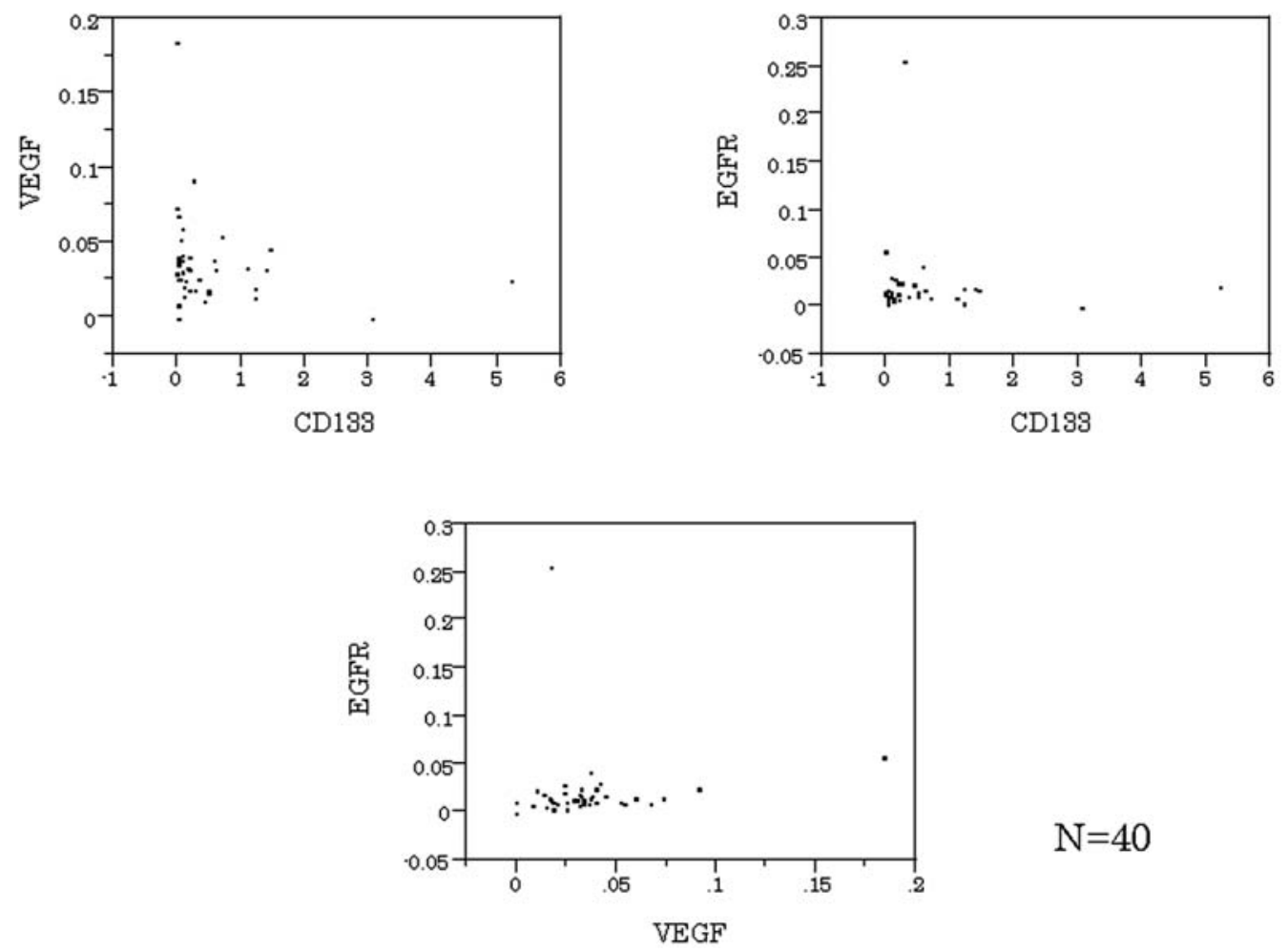

Figure 2. Correlations of CD133, VEGF and EGFR in post-CRT residual cancer cells. No significant correlations were observed in post-CRT FFPE specimens. 


\section{Post·CRT CD133, VEGF, and EGFR levels in residual rectal cancer and strormal cells}
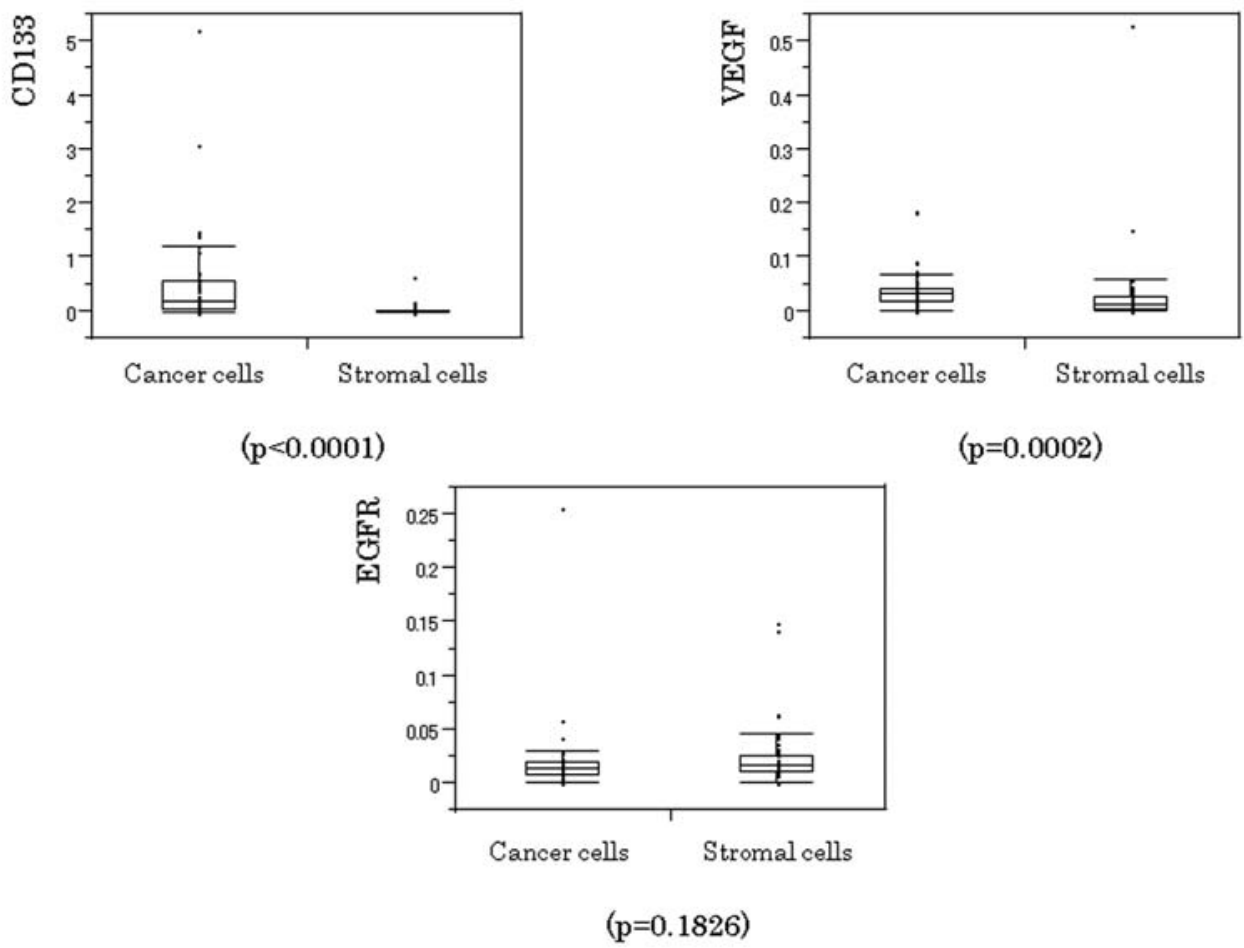

Figure 3. Post-CRT CD133, VEGF, and EGFR levels in residual rectal cancer and stromal cells. Post-CRT CD133 was significantly higher in residual cancer cells compared with those in stromal cells $(\mathrm{p}<0.0001)$. Post-CRT VEGF was also higher in residual cancer cells compared with those in stromal cells $(\mathrm{p}=0.0002)$. No significant difference in post-CRT EGFR levels was found between residual cancer and stromal cells. Box and whisker plots were used to summarize the distribution of mRNA level in residual cancer and stromal cells in post-CRT specimens. The horizontal line in the box represents the 50th (median) and the upper and lower lines of the box represent 75th and 25th quartiles, respectively. The whiskers indicate the range of the measurements.

Table III. Association of post-CRT CD133, VEGF, and EGFR with distant recurrence.

\begin{tabular}{lccc}
\hline Gene of target & Patients with distant recurrence $(\mathrm{n}=6)$ & Patients without distant recurrence $(\mathrm{n}=34)$ & $\mathrm{p}$-value \\
\hline CD133 & $0.833(0.048-2.143)$ & $0.156(0.0481-0.500)$ & $\mathbf{0 . 0 2}$ \\
VEGF & $0.035(0.017-0.056)$ & $0.032(0.018-0.039)$ & 0.97 \\
EGFR & $0.013(0.008-0.021)$ & $0.013(0.009-0.021)$ & 0.6 \\
\hline
\end{tabular}

Values of each target gene are expressed as median value (inter-quartile range). Bold type indicates a significant value.

Table IV. Expression of CD133, VEGF, and EGFR in matched pre-and post-CRT specimens.

\begin{tabular}{lccr}
\hline Gene of targets & Pre-CRT specimen $(\mathrm{n}=30)$ & Post-CRT specimens $(\mathrm{n}=30)$ & $\mathrm{p}$-value \\
\hline CD133 & $0.074(0.035-0.185)$ & $0.191(0.079-0.803)$ & $\mathbf{0 . 0 1 8 4}$ \\
VEGF & $0.413(0.172-0.875)$ & $0.032(0.018-0.043)$ & $\mathbf{< . 0 0 0 1}$ \\
EGFR & $0.404(0.047-1.128)$ & $0.014(0.010-0.021)$ & $\mathbf{0 . 0 0 0 2}$
\end{tabular}

Values of each target gene are expressed as median value (inter-quartile range). Bold type indicates a significant value.

used to identify each cut-off value of CD133 predictive of distant recurrence. A non-parametric ROC analysis showed that the best cut-off values of CD133 was 10.26. As shown in Fig. 4, patients with post-CRT CD133 above cut-off 
Disease-free survival according to the best cutoff value of post·CRT CD133

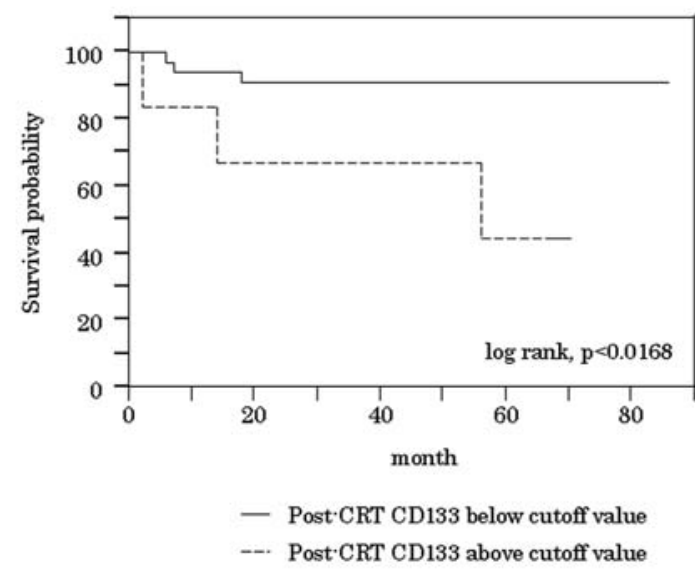

Figure 4. Disease-free survival according to the best cut-off value of postCRT CD133. Disease-free survival was evaluated using the Kaplan-Meier method. The log-rank test was used to compare the cumulative survival durations in the patient groups. Patients with post-CRT CD133 above cutoff value showed significantly worse disease-free survival $(\mathrm{p}<0.0168)$.

value showed significantly worse disease-free survival $(\mathrm{p}<0.0168)$.

Immunohistochemistry for CD133 in residual cancer cells after CRT. The minority of residual cancer cells had immunoreactive CD133 protein expression within entire residual tumor (Fig. 5).
CD133 was observed diffusely in cytoplasm of residual cancer cells and was also located at the apical/endoluminal surface (membranous) or cytoplasm (cytoplasmic) or both of residual cancer cells (Fig. 6).

\section{Discussion}

We showed the correlations between CD133 and angiogenesis-related genes such as VEGF and EGFR during CRT in rectal cancer. Positive correlations of CD133, VEGF and EGFR in pre-CRT rectal cancer were observed, but these correlations were not observed in post-CRT residual cancer cells. CD133 levels increased after CRT on paired specimens of each patient. In contrast, both VEGF and EGFR levels decreased after CRT.

There are several interpretations of these results. First of all, CRT may change these gene expression levels, resulting in the loss of correlations after CRT. Second, CRT may cause an imbalance between two putative populations within the tumor. A majority of tumor cells expressing VEGF and EGFR (considered as non-CSCs) may respond to CRT and subsequently shrink or disappear. In contrast, a very small population of cells expressing CD133 (considered as CSCs) may resist CRT and be left as residual cancer cells on postCRT specimens. Third, decreased expression levels of VEGF and EGFR may be partly due to the normalization of tumor hypoxia by CRT.

Although several factors may influence expression of CD133, VEGF, and EGFR during CRT, CD133 seems to be associated with tumor angiogenesis through VEGF or

Immunohistochemical CD133 expression in residual cancer cells after CRT
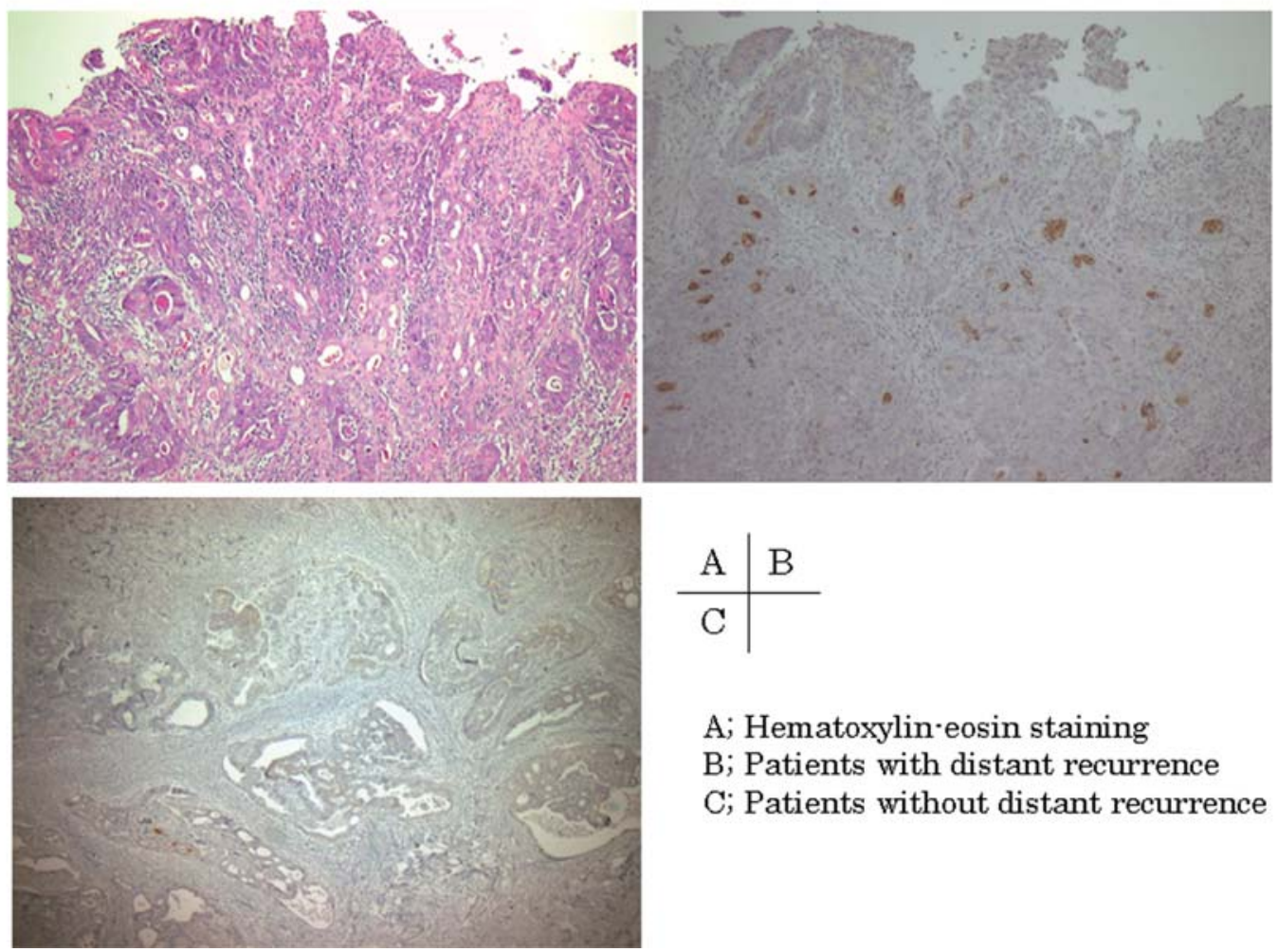

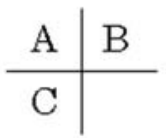
A; Hematoxylin-eosin staining
B; Patients with distant recurrence
C; Patients without distant recurrence

Figure 5. Immunohistochemical CD133 expression in residual cancer cells after CRT. The minority of residual cancer cells had immunoreactive CD133 protein expression within the entire residual tumor. 

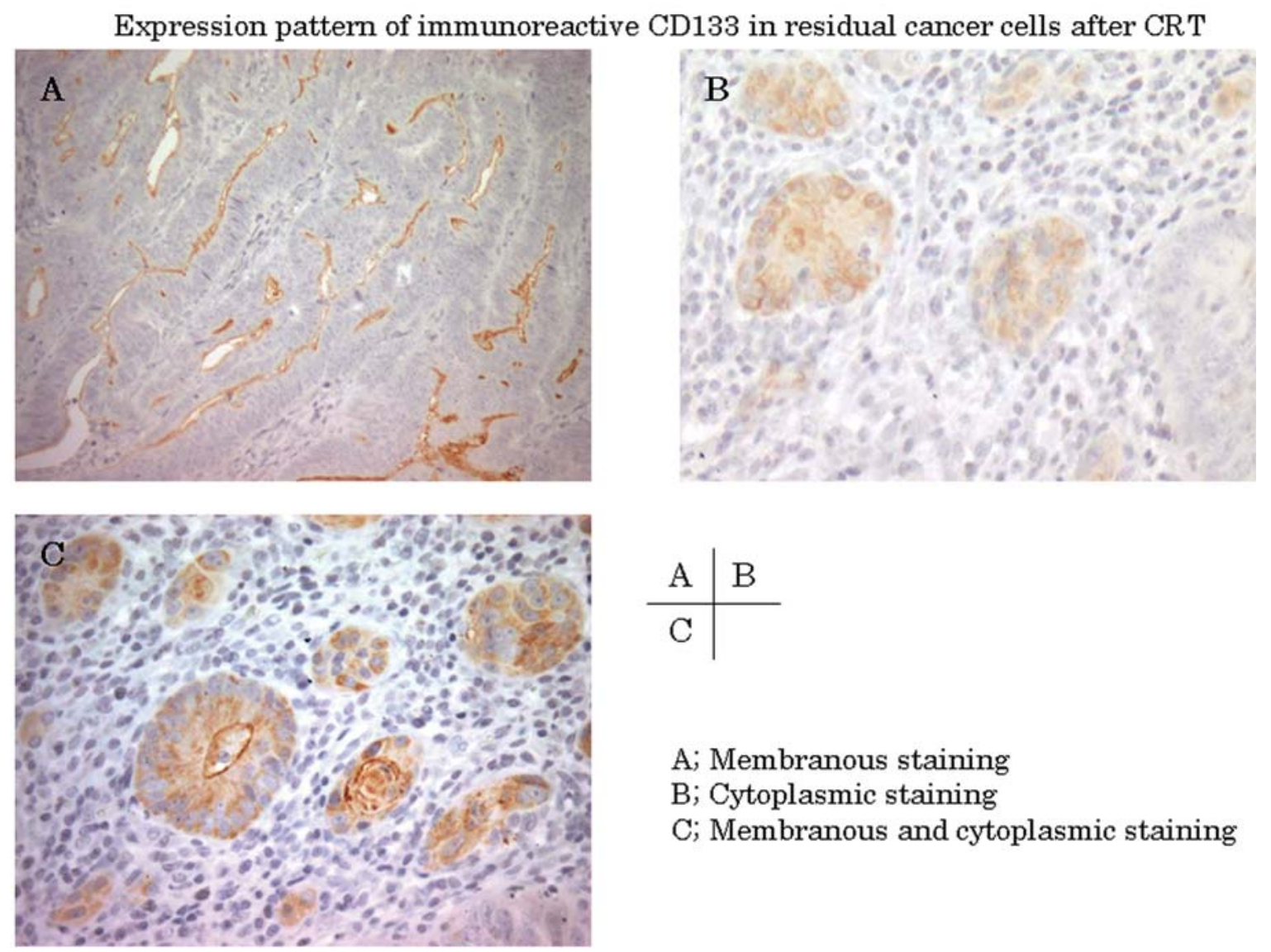

\section{A; Membranous staining \\ B; Cytoplasmic staining \\ C; Membranous and cytoplasmic staining}

Figure 6. Expression pattern of immunoreactive CD133 in residual cancer cells after CRT. CD133 was observed at the apical/endoluminal surface (A) or in cytoplasm (B) or both (C) of residual cancer cells.

EGFR. We also demonstrated that post-CRT CD133 was associated with distant recurrence and poor disease-free survival in rectal cancer, although we failed to provide any prognostic value of CD133 on pre-CRT specimens. Recently, the prognostic role of CD133 has been reported in pancreatic (11) and colorectal cancer (12). Prognostic value of TRG has been also reported in rectal cancer patients after CRT followed by TME (20). TRG is based on the percentage of residual cancer cells to stroma of entire tumor beds after CRT. The amount of residual cancer cells after CRT seems to be predictive of disease recurrence and survival in relation to CRT resistance. Our result showing the association of post-CRT CD133 with distant recurrence and poor survival appears to be consistent with a previous report on prognostic value of TRG.

Immunostaining of CD133 in residual cancer cells after CRT was shown in this study. CD133 positivity or immunohistochemical evaluation for CD133 remains controversial $(11,12,21)$. Recent studies propose that apical/endoluminal membranous CD133 staining was characteristic of welloriented, polarized and differentiated cells, while cytoplasmic CD133 staining was found in minor population of cells, suggesting that cancer cells with cytoplasmic CD133 staining may be indicative of putative CSCs $(21,22)$. Residual cancer cells represented membranous, cytoplasmic, or both of CD133 staining. As shown in Fig. 6, not all residual cancer cells expressed CD133. It is possible that post-CRT residual cancer cells may have relatively higher percentage of putative CSCs with CD133 expression. Post-CRT CD133 immunostaining may be useful in identifying rectal cancer patients with increased risk of distant recurrence after CRT if reliable and reproducible evaluation criteria for immunoreactive CD133 protein would be established.

However, data from this study should be interpreted with caution. The main limitation of this study was the relatively small number of patients $(n=40)$, including only six patients with distant recurrence. Large prospective trials will be needed to confirm the validity of the predictive value of post-CRT CD133 for distant recurrence.

In conclusion, CD133 in residual cancer cells after CRT may indicate a treatment resistant phenotype in putative CSCs. Elevated CD133, but not VEGF or EGFR, on FFPE specimens may be a predictive marker of distant recurrence and poor survival after preoperative CRT in rectal cancer. Expression analysis of CD133 in residual cancer may be useful for treatment stratification and clinical management in rectal cancer patients.

\section{References}

1. Bosset JF, Collette L, Calais G, et al: EORTC Radiotherapy Group Trial 22921. Chemotherapy with preoperative radiotherapy in rectal cancer. N Engl J Med 14: 1114-1123, 2006. 
2. Bujko K, Michalski W, Kepka L, et al: Polish Colorectal Study Group: association between pathologic response in metastatic lymph nodes after preoperative chemoradiotherapy and risk of distant metastases in rectal cancer: an analysis of outcomes in a randomized trial. Int J Radiat Oncol Biol Phys 67: 369-377, 2007.

3. Vermeulen L, Sprick MR, Kemper K, Stassi G and Medema JP: Cancer stem cells - old concepts, new insights. Cell Death Differ 15: 947-958, 2008

4. Shipitsin M and Polyak K: The cancer stem cell hypothesis: in search of definitions, markers, and relevance. Lab Invest 88: 459-463, 2008

5. Eyler CE and Rich JN: Survival of the fittest: cancer stem cells in therapeutic resistance and angiogenesis. J Clin Oncol 26: 2839-2845, 2008

6. Baumann M, Krause M and Hill R: Exploring the role of cance stem cells in radioresistance. Nat Rev Cancer 8: 545-554, 2008.

7. Ricci-Vitiani L, Lombardi DG, Pilozzi E, Biffoni M, Todaro M, Peschle $\mathrm{C}$ and De Maria R: Identification and expansion of human colon-cancer-initiating cells. Nature 445: 111-115, 2007.

8. O'Brien CA, Pollett A, Gallinger S and Dick JE: A human colon cancer cell capable of initiating tumour growth in immunodeficient mice. Nature 445: 106-110, 2007.

9. Hermann PC, Huber SL, Herrler T, et al: Distinct populations of cancer stem cells determine tumor growth and metastatic activity in human pancreatic cancer. Cell Stem Cell 1: 313-323, 2007.

10. Bao S, Wu Q, Sathornsumetee S, et al: Stem cell-like glioma cells promote tumor angiogenesis through vascular endothelial growth factor. Cancer Res 66: 7843-7848, 2006.

11. Maeda S, Shinchi H, Kurahara H, et al: CD133 expression is correlated with lymph node metastasis and vascular endothelial growth factor-C expression in pancreatic cancer. Br J Cancer 98: 1389-1397, 2008.

12. Horst D, Kriegl L, Engel J, Kirchner T and Jung A: CD133 expression is an independent prognostic marker for low survival in colorectal cancer. Br J Cancer 99: 1285-1289, 2008.
13. Galizia G, Lieto E, Ferraraccio F, et al: Determination of molecular marker expression can predict clinical outcome in colon carcinomas. Clin Cancer Res 10: 3490-3499, 2004.

14. Galizia G, Lieto E, Ferraraccio F, et al: Prognostic significance of epidermal growth factor receptor expression in colon cancer patients undergoing curative surgery. Ann Surg Oncol 13: $823-835,2006$

15. Ciardiello F, Troiani T, Bianco R, et al: Interaction between the epidermal growth factor receptor (EGFR) and the vascular endothelial growth factor (VEGF) pathways: a rational approach for multi-target anticancer therapy. Ann Oncol 17: 109-114, 2006

16. Tabernero J: The role of VEGF and EGFR inhibition: implications for combining anti-VEGF and anti-EGFR agents. Mol Cancer Res 5: 203-220, 2007.

17. Sobin LH and Fleming ID: TNM Classification of Malignant Tumors, fifth edition (1997). Union Internationale Contre le Cancer and the American Joint Committee on Cancer. Cancer 80: 1803-1804, 1997.

18. Dworak O, Keilholz L and Hoffmann A: Pathological features of rectal cancer after preoperative radiochemotherapy. Int J Colorectal Dis 12: 1219-1223, 1997.

19. Bijwaard KE, Aguilera NS, Monczak Y, Trudel M, Taubenberger JK and Lichy JH: Quantitative real-time reverse transcriptionPCR assay for cyclin D1 expression: utility in the diagnosis of mantle cell lymphoma. Clin Chem 47: 195-201, 2001.

20. Rödel C, Martus P and Papadoupolos T, et al: Prognostic significance of tumor regression after preoperative chemoradiotherapy for rectal cancer. J Clin Oncol 23: 8688-8696, 2005.

21. Immervoll H, Hoem D, Sakariassen PØ, Steffensen OJ and Molven A: Expression of the 'stem cell marker' CD133 in pancreas and pancreatic ductal adenocarcinomas. BMC Cancer 8: 48, 2008.

22. Mizrak D, Brittan M and Alison MR: CD133: molecule of the moment. J Pathol 214: 3-9, 2008. 